\title{
Metastatic lung cancer presenting as cutaneous nodules
}

\author{
Daniel Weinberg MBChB MPhil, Akheel A. Syed MBBS PhD
}

- Cite as: CMAJ 2017 July 24;189:E966. doi: 10.1503/cmaj.161519

A 68-year-old woman who had recently stopped smoking presented to the emergency department; she had experienced chest-wall pain for a few days, along with breathlessness and dry cough for a few weeks. On examination, she had three cutaneous lesions (Figure $1 \mathrm{~A})$ and a monophonic wheeze with reduced air entry at the left lung base. Chest x-ray (Figure 1B) and computed tomography (Figure $1 \mathrm{C}$ ) were consistent with a left upper lobe mass and metastases in the skin, adrenal glands and vertebrae. Magnetic resonance imaging (MRI) confirmed vertebral metastases (Figure 1D). Biopsy from a cutaneous lesion showed poorly differentiated carcinoma. A clinical diagnosis of metastatic lung cancer was made. The patient received palliative radiotherapy to the left lung and chest-wall metastasis only, as the cutaneous metastasis in this position was just above the breast tissue.

Cutaneous metastasis from internal cancers is uncommon. A meta-analysis that included more than 20000 patients with cancer reported an overall rate of $5.3 \%$ for all cancers. ${ }^{1}$ A 25 -year singlecentre United States Cancer Registry study of 401 patients with skin metastases found that breast cancer was the most common primary cancer (32.7\%), followed by bronchus and lung cancer (13.2\%). ${ }^{2}$ Other studies have found similar associations. ${ }^{3,4}$ The chest is the most common site of cutaneous metastasis and pain the most common symptom. ${ }^{2,5}$ Primary sarcomas, muscle lymphomas and benign diseases - including muscle hemangiomas, intramuscular ganglia and myxomas - must be considered in the differential diagnosis. MRI is useful in distinguishing these; biopsy is essential for definitive diagnosis. ${ }^{5}$ Treatment should be tailored to the patient's age, global health status, and nature and extent of the primary tumour, with surgical resection being reserved for patients with better prognosis, isolated lesions and a long disease-free interval. ${ }^{5}$
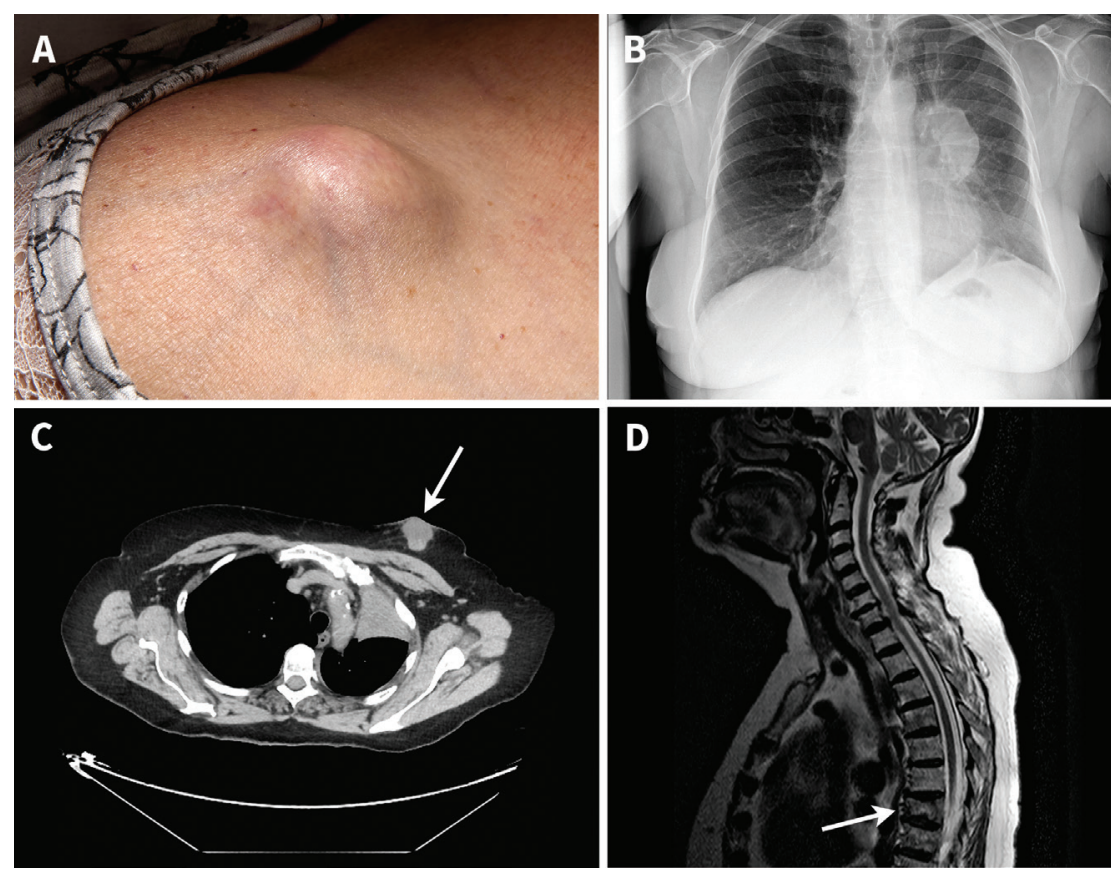

Figure 1: Advanced metastatic lung cancer in a 68-year-old woman, presenting with a cutaneous metastatic nodule $(A)$ on the right shoulder measuring $3 \mathrm{~cm}$ across; two other similar lesions were found overlying the left breast and the right flank. Biopsy from the latter showed poorly differentiated carcinoma. Chest x-ray (B) showed a left hilar shadow that had been absent on a previous chest $x$-ray eight months earlier. Computed tomography (C) showed infiltrative consolidation in the left lung and a cutaneous lesion in the left chest wall (arrow). Magnetic resonance imaging (D) showed multiple vertebral metastases and pathological fracture of T7 vertebra (arrow).

\section{References}

1. Krathen RA, Orengo IF, Rosen T. Cutaneous metastasis: a meta-analysis of data. South Med J 2003;96:164-7.

2. Wong CY, Helm MA, Helm TN, et al. Patterns of skin metastases: a review of 25 years' experience at a single cancer center. Int J Dermatol 2014;53:56-60.

3. Mollet TW, Garcia CA, Koester GK. Skin metastases from lung cancer. Dermatol Online J 2009;15:1.

4. Pajaziti L, Hapçiu SR, Dobruna S, et al. Skin metastases from lung cancer: a case report. BMC Res Notes 2015;8:139.

5. Perisano C, Spinelli MS, Graci C, et al. Soft tissue metastases in lung cancer: a review of the literature. Eur Rev Med Pharmacol Sci 2012;16:1908-14.

\section{Competing interests: None declared.}

This article has been peer reviewed.

The authors have obtained patient consent.
Affiliations: Department of Medicine (Weinberg, Syed), Salford Royal NHS Foundation Trust, Salford, UK; Faculty of Biology, Medicine \& Health (Syed), The University of Manchester, Manchester, UK

Correspondence to: Daniel Weinberg, Weinberg@doctors.org.uk 